\title{
Transit Frequency Optimization in Bi-modal Networks Using Differential Evolution Algorithm
}

\author{
Mehmet Metin MUTLU ${ }^{1}$ \\ İlyas Cihan AKSOY ${ }^{2}$ \\ Yalçın ALVER ${ }^{3}$
}

\begin{abstract}
This study proposes a bi-level optimization model for the transit frequency setting problem in bi-modal networks. The objective of the upper-level problem is to obtain a solution set of bus line frequencies that provide the minimum total travel cost of the car and bus users. Differential Evolution (DE) algorithm is employed in the upper-level model to determine the optimal headways for a given route structure. The lower-level model is a congested multimodal user equilibrium assignment model, which considers the interactions of car and bus flows, for determining joint mode/route preferences of the network users, which considers the interactions of car and bus flows. The developed model is tested on Mandl's benchmark network to evaluate its performance and applicability. The comparative experiments demonstrate that the proposed model leads to reductions in transportation costs. Also, the result of numerous optimization runs shows that DE performs well in finding similar frequency sets in independent optimizations.
\end{abstract}

Keywords: Transit frequency setting problem, bus frequency optimization, multi-modal assignment, differential evolution algorithm, bi-level optimization.

\section{INTRODUCTION}

Increasing population and urbanization, along with economic development, lead to higher mobility needs and transportation demand, especially in developing countries, such as Turkey. Commonly, the main policy of decision-makers is to mitigate transportation-related problems emerging due to increasing demand and meeting this demand by public transportation. However, unplanned public transit services may lead to insufficient supply

\footnotetext{
Note:

- This paper was received on May 11, 2021 and accepted for publication by the Editorial Board on January 11, 2022.

- Discussions on this paper will be accepted by November 30, 2022.

- https://doi.org/10.18400/tekderg.935950

1 Department of Civil Engineering, Aydın Adnan Menderes University, Aydın, Turkey metin.mutlu@adu.edu.tr - https://orcid.org/0000-0003-0008-8279

2 Department of Civil Engineering, Karamanoğlu Mehmetbey University, Karaman, Turkey icihanaksoy@kmu.edu.tr - https://orcid.org/0000-0002-4256-8222

3 Department of Civil Engineering, Ege University, İzmir, Turkey yalcin.alver@ege.edu.tr - https://orcid.org/0000-0002-9833-4505
} 
causing high travel costs or oversupply causing high operational costs. Furthermore, it can increase private transportation travel times by discouraging the use of public transit. On the other hand, a well-planned transit system can enhance transportation system for both public and private transportation.

Public transit networks can be improved through measures such as redesigning bus line routes, optimizing operational decisions such as bus line frequency setting, and traffic control measures such as allocating road network lanes like exclusive bus lanes. Optimal transit operation planning is a four-stage process, namely, network route design, timetable development, vehicle scheduling, and crew scheduling [1]. All stages should be handled simultaneously to obtain the optimum design. However, it is not possible with modern computing technology due to complex nature of these kind of problems [2]. Accordingly, each stage is usually solved sequentially [3].

Urban Transportation Network Design Problems are usually formulated as bi-level problems to allow considering passengers' reactions in network design decisions. The bi-level transportation network optimization problem is generally an NP-hard problem [4] commonly characterized by inherent non-convexity [5]. Due to the complexity of the problem, metaheuristics were proposed for network design problems in the literature for obtaining nearly global optimal solutions with high computation speeds at the expense of solution accuracy $[6,7]$.

Total user travel cost and operator cost minimization are frequently used objective functions for transit network design problems. It is possible to satisfy these objectives in a transit network solely by optimizing bus frequencies, which is studied under Transit Network Frequency Setting Problems (TNFSP).

There are numerous studies conducted related to TNFSP using heuristic approaches for mono-modal networks. [8] is one of the early studies adopting the bi-level model approach, in which the frequency setting problem is handled using a projected sub-gradient algorithm in the upper level with the aim of minimizing the total travel time and waiting times. The proposed model is performed in the transit networks of Stockholm (Sweden), Winnipeg (Canada), and Portland (USA). The study of [9] determines the line frequencies using a basic heuristic minimization algorithm, and implementing the proposed model to a small-size network. [10] proposes a frequency setting model to maximize demand under frequency value and fleet size constraints utilizing a gradient projection method. [11] determines both optimal frequencies and optimal bus sizes to minimize the sum of the total user cost and operator cost. The frequencies are determined using the Hooke-Jeeves algorithm, while the congested transit assignment is performed using ESTRAUS simulation software. In [12], a frequency setting model that minimizes total travel time of all users and required fleet size for the operators by Tabu Search is tested on a real-size network for morning-peak and offpeak periods. [13] determines frequencies by two different frequency determination methods, named optimum frequency and demand-based frequency methods, on the routes obtained by Ant Colony Optimization. Lastly, [14] develops a frequency setting model based on a novel objective function with the aim of decreasing the spread of COVID-19 derived from crowding at transit stops. The model aims to minimize the total infection risk at the stops, under a limited fleet size, employing the Differential Evolution Algorithm (DE). 
The studies presented above adopt the mono-modal assignment approach. This assumption is considered applicable for the networks where nonsignificant changes occur on the network since the minor improvements are unlikely to lead to user mode shifts between transit and private cars. However, if significant changes occur on the network, the network should be designed in consideration of elastic demand; thus, network design models should utilize multi-modal assignment. There are several transit frequency setting studies regarding the multi-modal assignment in the literature. [15] minimizes the weighted sum of users' costs, operator cost, and external cost using a heuristic algorithm, testing the model and the algorithm for a real-scale transit network. [16] determines the optimal frequency on a smallscale network with three transportation modes to minimize the total disutility using a stochastic user equilibrium assignment.

Reviewing the studies presenting the comparative performances of metaheuristics in different engineering fields; in [17], DE is compared to well-known metaheuristics, such as Genetic Algorithm (GA) and Simulated Annealing (SA), in the loop-layout design problem, and the findings show that DEA is superior to others; [18] addresses the performance comparison among the metaheuristics, Cuckoo-Search (CK), Particle Swarm Optimization (PSO), Differential Evolution (DE), and Artificial Bee Colony (ABC) by testing over 50 different benchmark functions. Statistical analysis demonstrates that the problem-solving success of $\mathrm{DE}$ and CK is relatively better than that of PSO and ABC; [19] presents a comparison of six meta-heuristic techniques to solve the multilevel thresholding problem: GA, PSO, DE, Ant Colony Optimization (ACO), SA, and Tabu Search (TS), and concluding that Differential Evolution is the most efficient in terms of the quality of the solution. Based on the results of these studies, in this study, DE is employed for performance evaluation in the problem of frequency optimization of transit lines in multi-modal networks.

In this study, a bi-level TNFSP model employing the Differential Evolution Algorithm (DE), an evolutionary metaheuristic algorithm, for optimizing frequencies in multi-modal networks is presented. The upper-level of the bi-level model is an optimization model that minimizes the total user cost by frequency setting, while the lower-level is a bi-modal assignment model that determines the transportation mode choice of the network users in addition to the route choices of private and public transportation modes. Therefore, the model proposed is suitable to be utilized as a decision support tool to optimize frequencies of public transportation lines considering the tradeoff between private car and public transportation use preferences, to minimize the costs of the users of both modes.

To our knowledge, there is no study employing DE for solving Transit Network Frequency Setting problems. The main contributions of this study are: proposing DE for a multi-modal TNFSP; examining the effect of different parameter values on the performance of DE in TNFSP; considering both private and public transportation modes in TNFSP for minimizing the total system cost by utilizing a multi-modal deterministic user equilibrium assignment in the lower-level model.

Section 2 of the study describes the upper-level and the lower-level models. Section 3 presents the numerical applications made on the benchmark network and discusses the results of the comparative experiments. Section 4 draws the conclusions of the study and highlights the possible directions for future research. 


\section{OPTIMIZATION MODEL}

In transportation planning, the effect of network design decisions on the performance of the transportation system and network users must be taken into consideration. Therefore, the transportation network design problem is usually formulated as a bi-level problem. The upper-level of the bi-level structure represents the decision-maker, whereas the lower-level represents travelers. This structure allows the decision-maker to take the reactions of travelers as well as network performance into consideration.

In this study, TNFSP is handled as a bi-level problem. The upper-level is a frequency optimization problem to minimize the total travel cost, and the lower-level is a multi-modal assignment problem.

\subsection{Upper-Level Model}

The objective of the optimization model is to determine the bus line frequency set, minimizing the total car and bus travel cost in the transportation system, which can be formulated as in (Eq. 1):

$\min \sum_{r s}\left(\sum_{k} q_{k}^{r s} \times t_{k}^{r s}+\sum_{\widehat{k}} \hat{q}_{\widehat{k}}^{r s} \times \hat{t}_{\widehat{k}}^{r s}\right) \forall r s \in W, \forall k \in K_{r s}, \forall \hat{k} \in \widehat{K}_{r s}$

where $W$ is the set of origin-destination (OD) pairs, $K_{r s}$ is the set of car paths on road network connecting OD pair $r s, \widehat{K}_{r s}$ is the set of transit paths on transit network connecting OD pair $r s, q_{k}^{r s}$ is the car flow on path $k, \hat{q}_{\hat{k}}^{r s}$ is the bus trip flow on path $\hat{k}, t_{k}^{r s}$ is the travel time on path $k, \hat{t}_{\widehat{k}}^{r s}$ is the travel time on the path $\hat{k}$.

Car path travel time is the sum of congested travel time of member links. The bus travel time of passengers consists of in-vehicle travel time and congested waiting time. Bus in-vehicle travel time is congested link travel time affected by both car and bus vehicle flows. Operator cost is not considered in this study for determining the optimal frequencies. The design constraint of the upper-level model regarding the bus fleet size $n_{b}$, imposes $0<n_{b} \leq n_{b}^{\max }$, where $n_{b}^{\max }$ is the maximum allowed fleet size, and $n_{b}$ is the minimum required number of buses to operate the transit network calculated by the frequency value and run time of each line.

Increased bus frequencies decrease waiting time, and consequently, total travel time decreases. Therefore, it is expected to result in shifts from private to public transportation. In this case, as the modal share of bus transportation increases, private car usage decreases, resulting in decreased car flows in the road network. As a result of lower car volumes, link travel times decrease. Subsequently, in a bi-modal transportation system, lower private car transportation costs induce shifts from public to private transportation. Decreasing bus frequencies, on the other hand, results in increased bus travel time and shifts from public to private transportation. In this case, increased link travel times due to high vehicle flow lead to an increase in travel times for both private and public transportation. An iterative process between the trip assignment model and optimization model is necessary to determine the optimal frequency set, minimizing the total travel cost. Due to the complex nature of the bilevel problems, it is not possible to determine the optimal solution using exact solution 
methods, especially in large-scale networks. Therefore, meta-heuristics are applied to obtain near-optimal solutions, especially for real-scale problems [20].

We employed DE for optimizing transit line frequencies in multi-modal networks in this study. DE, developed by [21], is an evolutionary approach to solve continuous-space problems and is considered to be one of the most prominent metaheuristics. DE consists of three main operators like genetic algorithms: mutation, crossover, selection operators.

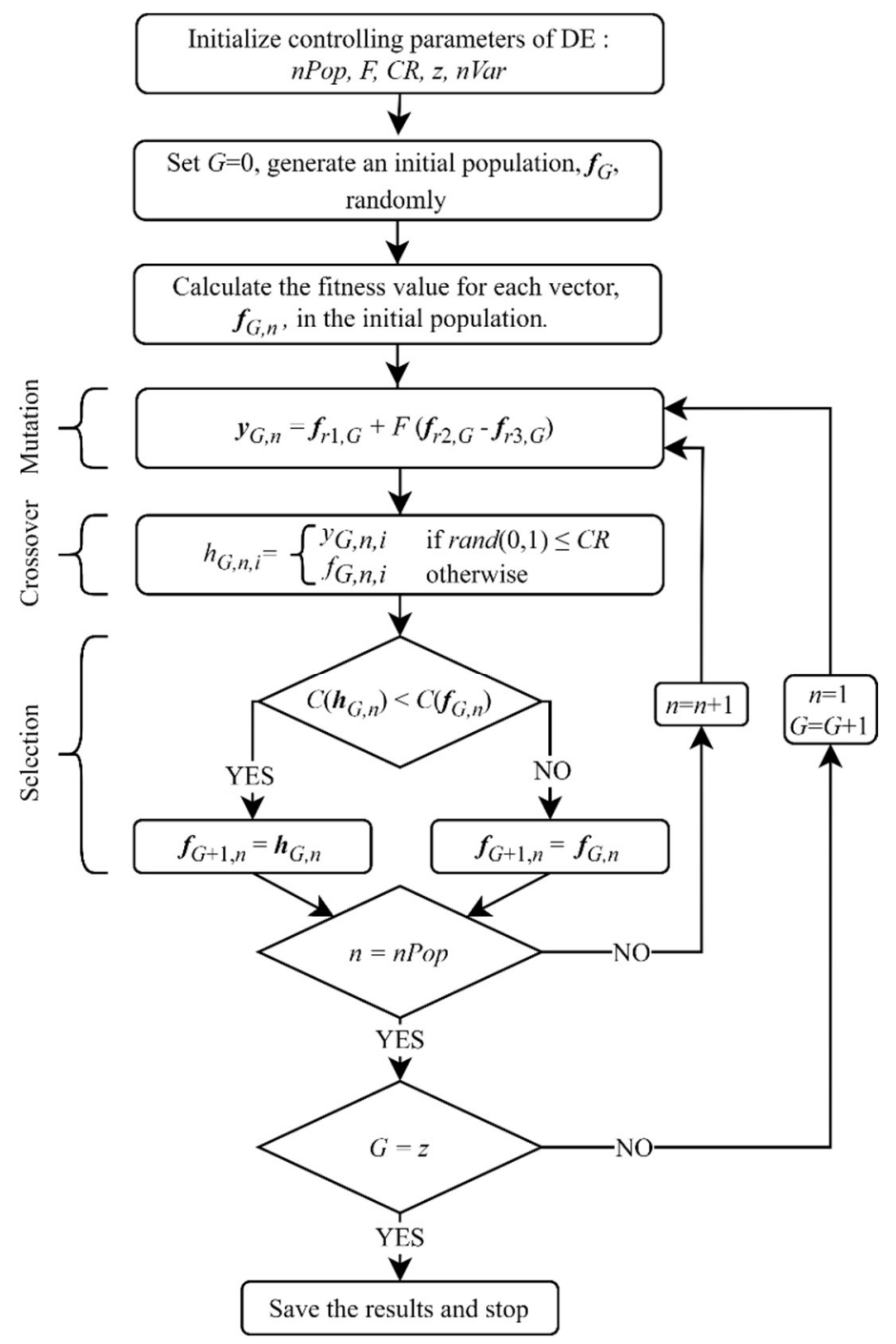

Figure 1 - The flowchart of traditional DE 
In the mutation operator, a mutant vector is created by weighting three random vectors (i.e., parent chromosomes) selected from the current population using the mutation constant, $F$, which controls the amplification of the differential variation. It should be noted that the selected three vectors must be different from each other. Following the generation of the mutant vector, a trial vector is generated by combining the target vector and the mutant vector with the help of the crossover constant, $C R$, that controls which genes of mutant and target vectors contribute to the trial vector. Finally, in the selection operator, a new target vector for the next generation is selected between the current target vector and the trial vector based on their fitness values. If the trial vector yields a smaller fitness value than the target vector value, the trial vector is replaced in the next generation; otherwise, the target vector is retained. The traditional DE steps are presented as a flowchart in Figure (1).

The algorithm that we used to solve the multi-modal frequency setting problem using DE is outlined as follows:

Step 0: Initialization. For each agent of the population with size nPop, generate a set of elements (frequencies for each line, nVar) $\boldsymbol{f}_{n}=\left\{f_{n, 1}, f_{n, 2}, \ldots, f_{n, \mathrm{nVar}}\right\}$, with random positions (frequency values) in the search space $\left(f_{\min }<f<f_{\max }\right)$.

Step 1: Calculation. Calculate cost (total travel time on the transportation system) for each frequency set, $\boldsymbol{f}_{n}$ by performing multi-modal trip assignment.

Step 2: For each agent of population, $n \in\{1,2, \ldots, \mathrm{nPop}\}$ :

Step 2.1: Mutation. Randomly select three different frequency set indices a, b and c from the population and generate mutation vector: $\boldsymbol{y}_{n}=\boldsymbol{f}_{a}+F\left(\boldsymbol{f}_{b}-\boldsymbol{f}_{c}\right)$ where $\boldsymbol{y}_{n}=$ $\left\{y_{n, 1}, y_{n, 2}, \ldots y_{n, n V a r}\right\}$ and mutation constant, $F \in[0,2]$.

Step 2.2: Crossover. Generate a random index $r \in\{1,2, \ldots, \mathrm{nVar}\}$ and for each element of an agent (frequency of line) generate a random number $R_{i} \in[0,1]$. For each element $i \in$ $\{1,2, \ldots, \mathrm{nVar}\}: h_{n, i}=\left\{y_{n, i}\right.$ if $R_{i} \leq C R$ or $i=r ; f_{n, i}$ otherwise $\}$, where crossover constant, $C R \in[0,1]$ is the crossover probability.

Step 2.3: Selection. Perform trip assignment with $\boldsymbol{h}_{n}$. Replace the corresponding element $\boldsymbol{f}_{n}$, with $\boldsymbol{h}_{n}$ if the solution is improved: $\boldsymbol{f}_{n}=\left\{\boldsymbol{h}_{n}\right.$ if $C\left(\mathbf{h}_{n}\right) \leq C\left(\boldsymbol{f}_{n}\right) ; \boldsymbol{f}_{n}$ otherwise $\}$

Step 3: Termination criterion. Stop if the maximum iteration number $z$ is reached and output the best solution; Otherwise, go to Step 2.

\subsection{Lower-Level Model}

Total travel cost calculation of the transportation system in the upper-level optimization model is possible through the output of the lower-level trip assignment model. The lowerlevel problem is a multi-modal network equilibrium assignment model with elastic demand in mode choice level, considering car and bus networks with flow interactions under the following assumptions: (1) All network users are assumed to choose the path minimizing their travel cost and transit network users are assumed to board the first arriving bus in the attractive lines set. (2) Transit stops are used as zones where demand originates and terminates. (3) Walking links are not included in the network; therefore, the assignment model does not allow passengers to walk between stops (4) All bus lines are assumed to have 
the same in-vehicle travel times while passing through the same itineraries. (5) Road network trip costs are calculated using the BPR link cost function, which does not consider the costs occurring at intersections. Thus, the assignment model assumes that congestions occur only on links and there are no delays caused by spillback queues and traffic lights, etc. (6) For the sake of simplicity, the car occupancy rate is assumed 1 passenger/car. (7) It is assumed that all travelers have access to a car, and therefore all public transportation users are considered choice riders in this study.

The assignment model acquires a solution that both car and transit flows to satisfy the deterministic user equilibrium criterion. The modal distributions are calculated using a logit type mode choice function (Eq. 2). The mode choice function employs the minimum travel costs of modes between OD pairs as parameters, and road network link travel time is assumed to follow the Bureau of Public Roads (BPR) function (Eq. 3), including bus vehicle flows considering passenger car equivalency.

$$
q_{r s}=\bar{q}_{r s} \frac{1}{1+e^{\theta\left(u_{r s}-\hat{u}_{r s}-\Psi_{r s}\right)}} \forall r s \in W
$$

where $q_{r s}$ is the car mode demand between OD pair $r s, \bar{q}_{r s}$ is total travel demand between $r s, u_{r s}$ is the cost of the shortest path between $r s$ on the road network, $\hat{u}_{r s}$ is the cost of the shortest path between $r s$ on transit network, $\Psi_{r s}$ is the car preference parameter.

$t_{a}=t_{a}^{0}\left[1+\alpha\left(\frac{x_{a}+x_{a, b u s} \times \mathrm{PCE}_{\mathrm{bus}}}{c_{a}}\right)^{\beta}\right] \forall a \in A$

where $t_{a}$ is congested link travel cost of link $a, t_{a}^{0}$ is the free-flow travel time of $a, x_{a}$ is car flow on $a, x_{a, b u s}$ is bus vehicle flow on $a, \mathrm{PCE}_{\text {bus }}$ is passenger car equivalency factor of bus, $c_{a}$ is the practical capacity of $a, \alpha$ and $\beta$ are calibration parameters.

Bus mode travel times are calculated utilizing a BPR-like function, given in Eq. 4, representing the effect of increased waiting time at stops due to congestion, as proposed by [22], in a transit network converted from line-segment representation to route-section representation as a proper method for handling common lines problem. It is possible to solve transit assignment problems similar to road network assignment problems utilizing routesection networks. The route section between a node pair consists of attractive lines that are a subset of bus lines that passengers can travel between the same node pair. In the route-section representation, nodes generally represent bus stops while links represent the route sections.

In this study, determining attractive lines to be included in each route section is based on the minimization of expected travel time as proposed in [23], and it is assumed that all lines passing through a road link have the same in-vehicle travel time. Consequently, all lines passing through the same road link sequence between a node pair are associated with a route section as attractive lines.

$\hat{t}_{s}=\bar{t}_{s}+\left(\frac{\widehat{\alpha}}{f_{s}}\right)+\hat{\beta} \times\left(\frac{\hat{x}_{s}+\tilde{x}_{s}}{\hat{C}_{s}}\right)^{\hat{n}} \forall s \in S$ 
where $\hat{t}_{s}$ is the total congested travel cost of route section $s, \bar{t}$ is the in-vehicle travel time of bus trips on $s$ which depends on vehicle flows on corresponding road network link set $\mathrm{A}, f$ is total frequencies of lines contained in $s, \hat{\alpha}$ is the calibration parameter for non-congested wait time at the stop, $\hat{x}_{s}$ is the number of passengers waiting for lines contained in $s, \tilde{x}_{s}$ is the total number of competitive passengers of $s$, who wait for other route sections that use lines contained in $s$ in the same stop, and passengers boarding the lines contained in $s$ at a node before the origin node of $s$ and alighting after, $\hat{C}_{s}$ is the practical capacity of $s$, which is the total capacity of the lines it contains, $\hat{\beta}$ is the calibration parameter for congested wait time at the stop.

Flow interaction between road and transit network links due to shared lane usage together with flow interaction on transit network due to competitive flows in transit network results in an asymmetric cost function in the assignment model employed in this study. Therefore, the assignment problem can be solved using the diagonalization method, which is commonly used for trip assignment problems with asymmetric cost functions due to non-symmetric link flow interactions [24,25]. Diagonalization is an iterative method that involves diagonalization of link cost functions to fix cross-link effects by fixing all arguments of a link other than its own flow in each iteration to solve a sub-problem [26]. The sub-problem solved at each iteration of the diagonalization algorithm using Frank and Wolfe Algorithm [27] for the multi-modal assignment problem presented in this study is formulated as:

$$
\begin{aligned}
& \min \tilde{z}^{(n)}=\sum_{a} \int_{0}^{x_{a}} \mathrm{t}_{a}\left(\omega, x_{a, b u s}^{(n)}\right) d \omega+\sum_{s} \int_{0}^{\hat{X}_{s}} \hat{\mathrm{t}}_{s}\left(\omega, \widetilde{\boldsymbol{x}}_{s}^{(n)}, \boldsymbol{x}_{s, \text { car }}^{(n)}\right) d \omega+ \\
& \sum_{r s} \int_{0}^{\hat{q}_{r s}}\left(\frac{1}{\theta} \ln \frac{\omega}{\bar{q}_{r s}-\omega}+\Psi_{r s}\right) d \omega
\end{aligned}
$$

subject to

$$
\begin{aligned}
& \sum_{k} X_{k}^{r s}=\bar{q}_{r s}-\hat{q}_{r s} \quad \forall r s ; \\
& \sum_{\hat{k}} \hat{X}_{\widehat{k}}^{r s}=\hat{q}_{r s} \quad \forall r s ; \\
& X_{k}^{r s}, \hat{X}_{\hat{k}}^{r s} \geq 0 \quad \forall k, \hat{k}, r s
\end{aligned}
$$

Equation (5a) is the objective function of the assignment problem composed of three terms. The first term is the sum of the integrals of the road network link cost functions to satisfy the road network user equilibrium criterion where $t_{a}$ is the road link cost function, $(n)$ is the iteration number and $x_{a, b u s}^{(n)}$ is the bus vehicle flow on the link at the $n^{\text {th }}$ iteration. The second term is the sum of the integrals of the route section network link cost functions to satisfy the transit network user equilibrium criterion where $\hat{t}_{s}$ is the cost function of route section $s, \widetilde{\boldsymbol{x}}_{s}^{(n)}$ is the bus competitive flow vector of $s$ at the $n^{\text {th }}$ iteration, and $\boldsymbol{x}_{s, \text { car }}^{(n)}$ is the vector of car flows on the road network links that $s$ incorporates, affecting the in-vehicle cost of $s$ at the $n^{\text {th }}$ iteration. The last term is the sum of integrals of the inverse of the logit demand function for each OD pair, ensuring the equilibrium criterion for the car and transit demand share as proposed by [26]. Equations (5b) and (5c) are flow conservation constraints for the road network and route section network, respectively, and equation (5d) is the nonnegativity of 
flow constraint for both networks, where $X_{k}^{r s}$ is the flow on path $k$ between OD pair $r s$ on the road network, $\hat{X}_{\hat{k}}^{r s}$ is the flow on path $\hat{k}$ between $r s$ on the route section network.

\section{TEST NETWORK APPLICATION}

The proposed model is tested on Mandl's Swiss network, which has been used by several authors in the literature [28-30]. The test network consists of 15 nodes and 42 unidirectional links. The line routes to be used are obtained from the study of [29]. Automatically generated route-section network with the given road network and bus lines consists of 282 unidirectional links. The original demand is increased by a factor of 2 considering the modal choice since the original network is used solely for the transit demand.
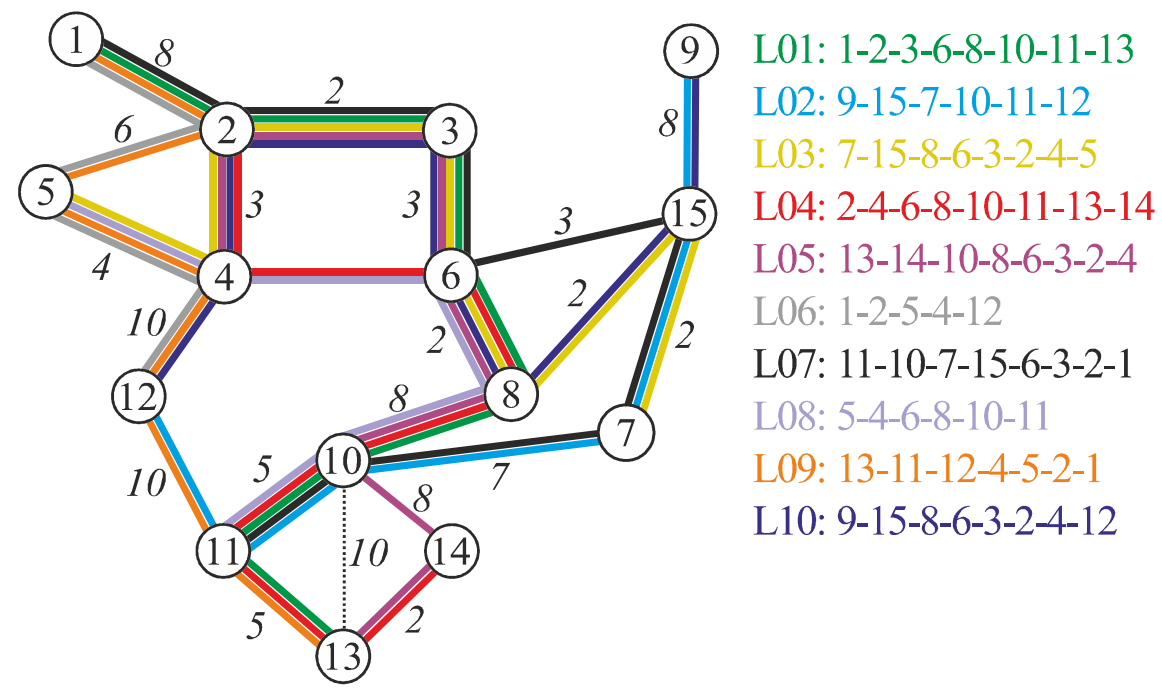

Figure 2 - Test network and bus lines

In Figure 2, the bus line routes of the test network are represented by different colors. The free-flow travel times of each link independent of directions, are indicated on the relevant link in minutes. The node sequence of each route in one direction is also presented on the right of the network. The OD matrix of the total trip demand in the analysis period used in numerical applications is given in Table 1 .

In the transit network, assuming that the operator has a limited fleet size, a fleet size constraint is incorporated into the optimization model. Fleet size and bus capacities are assumed 300 buses and 100 passengers, respectively. For all links in the road network, practical capacities are $800 \mathrm{veh} / \mathrm{h}$. Cost function parameters of the assignment model are defined as $\theta=0.3$, $\Psi_{r s}=0, \alpha=0.15, \beta=4, \mathrm{PCE}_{\mathrm{bus}}=3, \hat{\alpha}=0.5, \hat{\beta}=1, \hat{n}=1$. Allowed minimum and maximum values of line frequency are defined as 1 and 60 , respectively. 
Transit Frequency Optimization in Bi-modal Networks Using Differential ...

Table 1 - Node-to-node trip demand matrix used in test network application

\begin{tabular}{cccccccccccccccc}
\hline OD & $\mathbf{1}$ & $\mathbf{2}$ & $\mathbf{3}$ & $\mathbf{4}$ & $\mathbf{5}$ & $\mathbf{6}$ & $\mathbf{7}$ & $\mathbf{8}$ & $\mathbf{9}$ & $\mathbf{1 0}$ & $\mathbf{1 1}$ & $\mathbf{1 2}$ & $\mathbf{1 3}$ & $\mathbf{1 4}$ & $\mathbf{1 5}$ \\
\hline $\mathbf{1}$ & 0 & 800 & 400 & 120 & 160 & 300 & 150 & 150 & 60 & 320 & 60 & 50 & 70 & 0 & 0 \\
\hline $\mathbf{2}$ & 800 & 0 & 100 & 240 & 40 & 360 & 180 & 180 & 30 & 260 & 40 & 20 & 20 & 10 & 0 \\
\hline $\mathbf{3}$ & 400 & 100 & 0 & 80 & 120 & 360 & 180 & 180 & 30 & 90 & 40 & 20 & 20 & 10 & 0 \\
\hline $\mathbf{4}$ & 120 & 240 & 80 & 0 & 100 & 200 & 100 & 100 & 30 & 480 & 80 & 50 & 20 & 10 & 0 \\
\hline $\mathbf{5}$ & 160 & 40 & 120 & 100 & 0 & 100 & 50 & 50 & 20 & 240 & 40 & 30 & 10 & 0 & 0 \\
\hline $\mathbf{6}$ & 300 & 360 & 360 & 200 & 100 & 0 & 200 & 200 & 60 & 1760 & 120 & 30 & 30 & 20 & 0 \\
\hline $\mathbf{7}$ & 150 & 180 & 180 & 100 & 50 & 200 & 0 & 100 & 30 & 880 & 70 & 20 & 20 & 10 & 0 \\
\hline $\mathbf{8}$ & 150 & 180 & 180 & 100 & 50 & 200 & 100 & 0 & 30 & 880 & 70 & 20 & 20 & 10 & 0 \\
\hline $\mathbf{9}$ & 60 & 30 & 30 & 30 & 20 & 60 & 30 & 30 & 0 & 280 & 40 & 10 & 0 & 0 & 0 \\
\hline $\mathbf{1 0}$ & 320 & 260 & 90 & 480 & 240 & 1760 & 880 & 880 & 280 & 0 & 1200 & 500 & 1000 & 400 & 0 \\
\hline $\mathbf{1 1}$ & 60 & 40 & 40 & 80 & 40 & 120 & 70 & 70 & 40 & 1200 & 0 & 150 & 190 & 30 & 0 \\
\hline $\mathbf{1 2}$ & 50 & 20 & 20 & 50 & 30 & 30 & 20 & 20 & 10 & 500 & 150 & 0 & 140 & 0 & 0 \\
\hline $\mathbf{1 3}$ & 70 & 20 & 20 & 20 & 10 & 30 & 20 & 20 & 0 & 1000 & 190 & 140 & 0 & 90 & 0 \\
\hline $\mathbf{1 4}$ & 0 & 10 & 10 & 10 & 0 & 20 & 10 & 10 & 0 & 400 & 30 & 0 & 90 & 0 & 0 \\
\hline $\mathbf{1 5}$ & 0 & 0 & 0 & 0 & 0 & 0 & 0 & 0 & 0 & 0 & 0 & 0 & 0 & 0 & 0 \\
\hline
\end{tabular}

Algorithms used in this study were coded using MATLAB R2020a and carried out on a 64bit computer with an AMD Ryzen $536003.60 \mathrm{GHz}$ CPU and 16 GB RAM. The average duration of an optimization terminated at the $100^{\text {th }}$ iteration is 280 minutes.

Metaheuristic algorithms are sensitive to the values of the parameters. In problems with numerous local optima such as TNFSP, reaching optimal or near-optimal solutions may be difficult and time-consuming for the algorithms because of improper parameter values. Thus, $\mathrm{DE}$ is executed with the different combinations of parameter values to better show the effect of different parameter values on the performance of DE.

Parameters $F$ and $C R$ values are chosen from the sets $\{0.5,1.0,1.5,2.0\}$ and $\{0.2,0.4,0.6,0.8\}$, respectively, resulting in 16 combinations. Also, three replications for each combination are carried out to avoid statistical deviations, leading to 48 optimization runs in total. It is likely that the increase in nPop and $z$ values obtain better solutions; therefore, the effects of these parameters are not investigated in this study. nPop and the maximum iteration number are defined as 50 and 100, respectively. Table 2 demonstrates the results of 16 different parameter combinations, showing the average total system cost of five optimizations for each parameter combination.

Table 2 clearly shows that the best combination of parameter values is obtained in the combination $F=0.5$ and $C R=0.6$, with a cost of 901,757 . To demonstrate the stability of the algorithm, 30 optimizations are performed using the calibrated parameter values. The obtained frequency sets and the corresponding costs of 30 optimization runs are given in Table 3. 
Table 2 - The results of different combinations of parameter values (Average total system costs in $\mathrm{min}$ )

\begin{tabular}{|c|c|c|c|c|}
\hline$F$ & 0.2 & 0.4 & 0.6 & 0.8 \\
\hline 0.5 & 914,264 & 903,797 & 901,757 & 902,014 \\
\hline 1.0 & 908,573 & 913,523 & 912,798 & 909,029 \\
\hline 1.5 & 914,523 & 920,224 & 932,105 & 916,026 \\
\hline 2.0 & 930,134 & 926,153 & 941,997 & 949,893 \\
\hline
\end{tabular}

Standart Deviation $=14,885$, Coefficient of Variation $=0.016$ in 48 runs

Table 3 - The results of 30 solutions by the calibrated parameter values

\begin{tabular}{|c|c|c|}
\hline $\begin{array}{l}\text { Total System } \\
\text { Cost (min) }\end{array}$ & $\begin{array}{l}\text { Frequency values of the lines (runs/h) } \\
\qquad\left\{f_{1,} f_{2,} f_{3,} f_{4}, f_{5}, f_{6}, f_{7,} f_{8}, f_{9}, f_{10}\right\}\end{array}$ & $\begin{array}{l}\text { Frequency of Being } \\
\text { Obtained as a Solution }\end{array}$ \\
\hline 899,220 & $\{1.0,1.9,1.6,1.0,18.6,1.3,28.6,24.2,21.2,19.9\}$ & 1 \\
\hline 900,079 & $\{1.0,1.7,1.0,3.0,12.9,1.3,29.0,26.8,20.8,21.9\}$ & 4 \\
\hline 900,686 & $\{1.4,1.0,1.0,1.0,15.2,1.0,28.5,25.9,22.5,21.9\}$ & 1 \\
\hline 900,691 & $\{1.8,3.9,1.0,2.5,13.6,1.0,29.7,27.3,19.7,16.8\}$ & 2 \\
\hline 902,120 & $\{1.2,2.2,2.1,2.1,11.8,1.1,29.5,29.4,19.9,18.5\}$ & 2 \\
\hline 902,309 & $\{1.1,1.0,1.0,1.0,13.0,1.1,32.3,27.5,18.5,18.0\}$ & 2 \\
\hline 902,509 & $\{1 \cdot 1,1 \cdot 0,1.0,1.2,15 \cdot 5,1.0,30 \cdot 2,24 \cdot 0,21.7,19.5\}$ & 1 \\
\hline 902,795 & $\{1.0,4.6,1.4,1.2,21.3,1.2,27.8,21.6,19.2,20.8\}$ & 4 \\
\hline 902,845 & $\{1.0,3.9,1.3,5.2,18.8,1.0,29.8,17.9,20.0,19.6\}$ & 1 \\
\hline 902,866 & $\{1.0,12.1,3.3,1.0,19.1,1.0,29.4,25.7,17.5,5.3\}$ & 2 \\
\hline 902,991 & $\{1.0,3.6,1.1,1.0,17.5,1.0,30.2,26.7,21.5,12.0\}$ & 1 \\
\hline 903,329 & $\{1.0,1.0,1.2,4.4,14.0,1.0,30.8,24.1,17.5,25.1\}$ & 1 \\
\hline 905,035 & $\{1.0,13.1,5.4,1.0,19.2,1.5,27.8,24.8,16.6,7.2\}$ & 2 \\
\hline 905,343 & $\{1.0,14.8,2.4,1.0,25.4,2.0,26.5,20.9,16.4,4.6\}$ & 1 \\
\hline 906,114 & $\{1.5,5.2,3.1,1.0,21.2,1.0,29.7,25.4,15.4,14.3\}$ & 2 \\
\hline 906,743 & $\{1.1,15.3,5.2,1.0,23.0,2.0,27.6,24.4,13.9,2.8\}$ & 1 \\
\hline
\end{tabular}

Standard Deviation $=1,921$, Coefficient of Variation $=0.0021$ in 30 optimizations

The statistical outputs given in the last row of both tables affirm the necessity of the calibration process for the parameter values. The best solution obtained is given in the first row of Table 3, with a total system cost of 899,220 . Network performance outputs, namely average travel times and modal share ratios, are calculated by running the combined mode choice assignment process using the frequency set obtained from the optimization. In the best 
solution, the average travel times of car and bus trips are $17.38 \mathrm{~min}$ and $11.49 \mathrm{~min}$ per user, respectively, and the transit modal share is $40 \%$. Figure 3 depicts the convergence process along 100 iterations for 30 runs by the calibrated parameter values. As can be seen, the algorithms complete the convergence process in approximately 40 iterations.

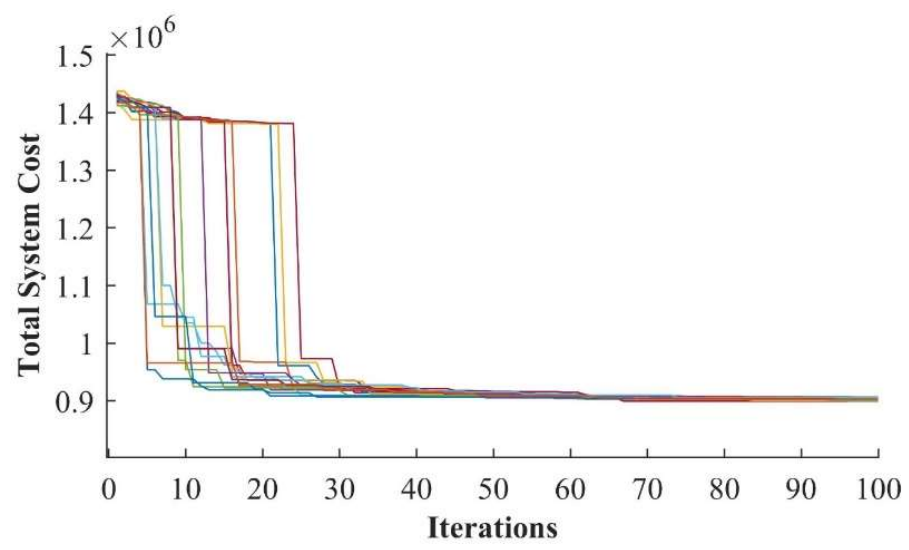

Figure 3 - Convergence of 30 solutions along with iterations

Firstly, the bus line frequencies are optimized using a model without a fleet size constraint. The results given in Table 4 show that minimum system cost is obtained with a fleet size of 339. Therefore, optimizing with a fleet size constraint greater than 339 buses is unnecessary since the same solution is expected to be obtained.

Table 4 - The results without fleet size constraint by the proposed model

\begin{tabular}{|c|c|c|c|c|}
\hline $\begin{array}{l}\text { Total } \\
\text { System } \\
\text { Cost } \\
(\text { min) }\end{array}$ & $\begin{array}{l}\text { Private } \\
\text { Car } \\
\text { Cost } \\
\text { (min) }\end{array}$ & $\begin{array}{c}\text { Public } \\
\text { Transportation } \\
\text { Cost (min) }\end{array}$ & $\begin{array}{l}\text { Fleet } \\
\text { Size }\end{array}$ & $\begin{array}{l}\text { Frequency values of the lines (runs/h) } \\
\qquad\left\{f_{1}, f_{2}, f_{3}, f_{4}, f_{5}, f_{6}, f_{7}, f_{8}, f_{9}, f_{10}\right\}\end{array}$ \\
\hline 874,568 & 504,033 & 370,534 & 339 & $\{1.0,1.1,1.0,2.0,18.8,1.1,40.8,35.8,26.2,36.4\}$ \\
\hline
\end{tabular}

In multi-modal networks with link flow interaction, the increase in the frequency of transit lines makes transit systems more attractive and leads to an increase in the use of transit systems. Thus, the congestion on the roads and the total system cost for all users are reduced. However, the further increase in frequency values of transit lines causes an increase in transit vehicle flow on the road links, triggering congestion for the vehicles of both modes. To prove this argument, following a simple assumption, the total system costs are calculated for the scenarios that all lines take the same frequency values between 1 and 60, as shown in Figure 4. The best frequency set is obtained using the frequency set of $18 \mathrm{runs} / \mathrm{h}$ for all lines with a minimum total system cost of 923,140 , and 446 buses are needed to operate the network. Further improvement in total system cost is possible by optimizing the frequency values of lines individually. 


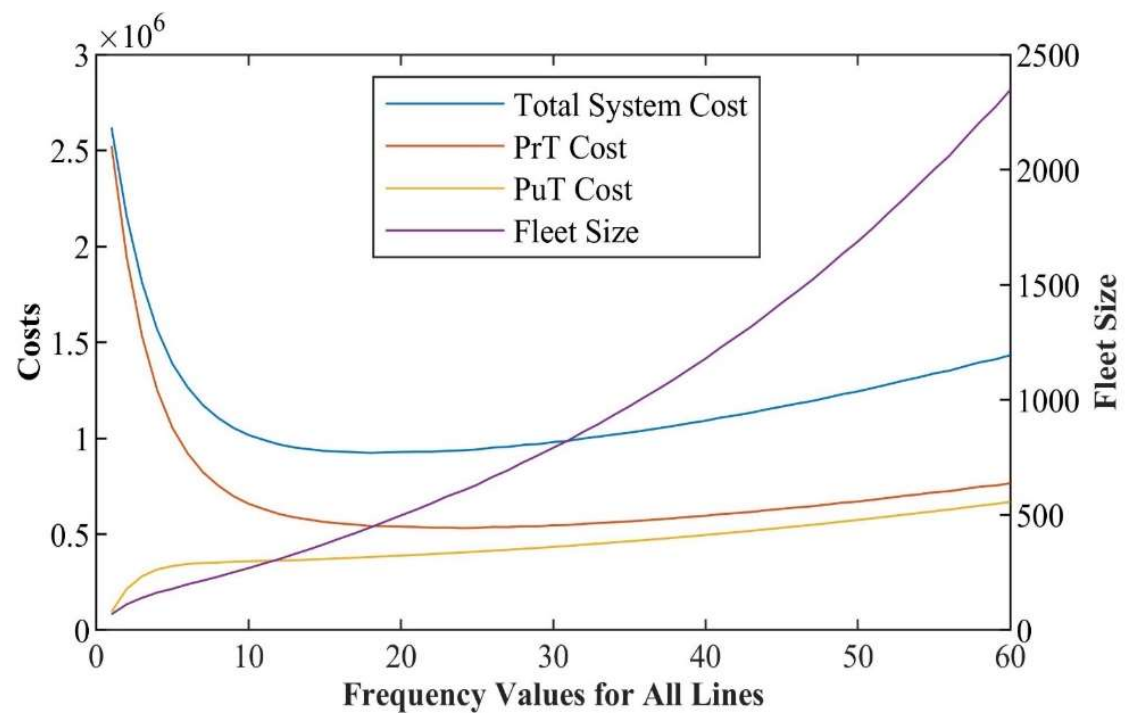

Figure 4 - Total system costs (min) for frequency sets in multi-modal networks

To assign the same frequency value to all lines, as shown in Figure 4, is a simple method; however, in order to demonstrate the stability and robustness of the proposed algorithm, we benefit from the solutions for comparison purposes. The frequency sets with 6,12 , and 15 runs/h for all lines require 199, 308, and 374 buses to operate the network, respectively. The optimization model is run with these fleet size constraints to compare the optimization model results with fixed-frequency calculation results in terms of private transportation and public transportation total user costs. The comparison results are given in Table 5. The reductions in total system costs for the optimizations with fleet size constraints of 199, 308, and 374 buses are $12 \%, 7 \%$, and $6 \%$, respectively.

Table 5 - The comparison between the results of the proposed model and the simple method

\begin{tabular}{|c|c|c|c|c|c|c|}
\hline $\begin{array}{l}\text { Fleet } \\
\text { Size } \\
\text { (bus) }\end{array}$ & $\begin{array}{l}\text { Reduction } \\
(\%)\end{array}$ & $\begin{array}{l}\text { Total } \\
\text { System } \\
\text { Cost } \\
(\mathrm{min})\end{array}$ & $\begin{array}{l}\text { Private } \\
\text { Car Cost } \\
\quad(\min )\end{array}$ & $\begin{array}{l}\text { Public } \\
\text { Trans. } \\
\text { Cost } \\
(\mathrm{min})\end{array}$ & $\begin{array}{l}\text { Public } \\
\text { Trans. } \\
\text { Share } \\
(\%)\end{array}$ & $\begin{array}{l}\text { Frequency values of the lines (runs/h) } \\
\qquad\left\{f_{1}, f_{2}, f_{3}, f_{4}, f_{5}, f_{6}, f_{7}, f_{8}, f_{9}, f_{10}\right\}\end{array}$ \\
\hline \multirow{2}{*}{199} & \multirow{2}{*}{-12} & $1,115,326$ & & 349,576 & 32 & $\{13.3,10.1,1.3,1.0,13.3,1.0,7.4,9.4,2.7,1.0\}$ \\
\hline & & $1,265,426$ & 920,639 & 344,787 & 28 & $\{6,6,6,6,6,6,6,6,6,6\}$ \\
\hline \multirow{2}{*}{308} & & 901,333 & 544,349 & 356,983 & 39 & $\{1.0,1.0,1.3,7.7,9.1,1.7,33.7,23 \cdot 0,21.0,18.2\}$ \\
\hline & & 967,510 & 605,094 & 362,416 & 37 & $\{12,12,12,12,12,12,12,12,12,12\}$ \\
\hline \multirow{2}{*}{374} & & 877,787 & 511,514 & 366,273 & 42 & $\{1.0,2.0,1 \cdot 2,2.2,16.2,1.0,37.2,34.7,25.1,30.6\}$ \\
\hline & & 933,608 & 563,192 & 370,415 & 40 & $\{15,15,15,15,15,15,15,15,15,15\}$ \\
\hline
\end{tabular}




\section{CONCLUSION}

In this study, we propose a bi-level optimization model utilizing DE for TNFSP in bi-modal networks. The objective is to minimize the total travel cost in the transportation system for both car and bus modes. The proposed model is calibrated and tested on a benchmark network. The optimization results are compared with a simple fixed-frequency cost calculation approach. Numerous optimization runs resulted in similar and consistent solutions with significant decreases in cost, showing that DE is applicable in TNFSPs. Also, the significant reductions in the total system cost obtained using the proposed model are shown as proof of the necessity of optimization models for determining bus line frequencies.

Multi-modal assignment results indicate that bus link travel times are significantly affected by car flows. Consequently, bus travel time is always higher than car travel time since bus travel time also includes waiting time, even in uncongested situations, as expected. Therefore, the use of exclusive bus lanes can be considered to achieve a competitive public transit network leading to an increased modal share of bus mode.

Bi-level models with meta-heuristics in the upper level require numerous repetitions of the lower-level model. Therefore, a computationally less expensive static assignment model is utilized in this study to ensure reasonable optimization durations. However, using static models compared to dynamic assignment models or microscopic simulation models may lead to less realism regarding trip costs, path choices, etc. Another limitation of this study is that all public transportation users are considered choice riders and have access to a car.

In future studies, taking captive riders into consideration will lead to a more realistic trip assignment model. Additionally, to develop a more realistic and more sustainable transit network design model, the proposed model will be improved by including the exclusive bus lane location decisions in transit network design.

\section{Acknowledgments}

The authors would like to thank two anonymous reviewers for their constructive comments that contributed to improve this paper.

\section{References}

[1] Ceder. A., Public Transit Planning and Operation: Modeling, Practice and Behavior, CRC Press, Boca Raton, USA, 2015.

[2] Magnanti, T. L., Wong, R. T., Network Design and Transportation Planning: Models and Algorithms. Transportation Science, 18(1), 1-55, 1984.

[3] Guihaire, V., Hao, J.-K. K., Transit network design and scheduling: A global review. Transportation Research Part A: Policy and Practice, 42(10), 1251-1273, 2008.

[4] Zhao, F., Large-Scale Transit Network Optimization by Minimizing User Cost and Transfers. Journal of Public Transportation, 9(2), 107-129, 2006.

[5] Yang, H., Bell, M. G. H., Models and algorithms for road network design: a review and some new developments. Transport Reviews, 18(3), 257-278, 1998. 
[6] Farahani, R. Z., Miandoabchi, E., Szeto, W. Y., Rashidi, H., A review of urban transportation network design problems. European Journal of Operational Research, 229(2), 281-302, 2013.

[7] Ibarra-Rojas, O. J., Delgado, F., Giesen, R., Munoz, J. C., Planning, operation, and control of bus transport systems: A literature review. Transportation Research Part B: Methodological, 77, 38-75, 2015.

[8] Constantin, I., Florian, M., Optimizing Frequencies in a Transit Network: A Nonlinear Bi-level Programming Approach. International Transactions in Operational Research, 2(2), 149-164, 1995.

[9] Gao, Z., Sun, H., Shan, L. L., A continuous equilibrium network design model and algorithm for transit systems. Transportation Research Part B: Methodological, 38(3), 235-250, 2004.

[10] Yoo, G. S., Kim, D. K., Chon, K. S., Frequency design in urban transit networks with variable demand: Model and algorithm. KSCE Journal of Civil Engineering, 14(3), 403-411, 2010.

[11] dell'Olio, L., Ibeas, A., Ruisánchez, F., Optimizing bus-size and headway in transit networks. Transportation, 39(2), 449-464, 2012.

[12] Giesen, R., Martinez, H., Mauttone, A., Urquhart, M. E., A method for solving the multi-objective transit frequency optimization problem. Journal of Advanced Transportation, 50 (8), 2323-2337, 2016.

[13] Gholami, A., Tian, Z., The comparison of optimum frequency and demand based frequency for designing transit networks. Case Studies on Transport Policy, 7(4), 698707, 2019.

[14] Mutlu, M. M., Aksoy, İ. C., Alver, Y., COVID-19 transmission risk minimization at public transportation stops using Differential Evolution algorithm. European Journal of Transport and Infrastructure Research, 21(1), 53-69, 2021.

[15] Gallo, M., D'Acierno, L., Montella, B., A multimodal approach to bus frequency design. $17^{\text {th }}$ International Conference on Urban Transport and the Environment, Pisa, Italy, 2011.

[16] Uchida, K., Sumalee, A., Watling, D., Connors, R., Study on Optimal Frequency Design Problem for Multimodal Network Using Probit-Based User Equilibrium Assignment. Transportation Research Record: Journal of the Transportation Research Board, 1923(1), 236-345, 2005.

[17] Nearchou, A. C., Meta-heuristics from nature for the loop layout design problem. International Journal of Production Economics, 101(2), 312-328, 2006.

[18] Civicioglu, P., Besdok, E., A conceptual comparison of the Cuckoo-search, particle swarm optimization, differential evolution and artificial bee colony algorithms. Artificial Intelligence Review, 39, 315-346, 2013. 
[19] Hammouche, K., Diaf, M., Siarry, P., A comparative study of various metaheuristic techniques applied to the multilevel thresholding problem. Engineering Applications of Artificial Intelligence, 23(5), 676-688, 2010.

[20] Miandoabchi, E., Daneshzand, F., Szeto, W. Y., Farahani, R. Z., Multi-objective discrete urban road network design. Computers \& Operations Research. 40(10), 24292449, 2013.

[21] Storn, R., Price, K.,. Differential Evolution - A Simple and Efficient Heuristic for Global Optimization over Continuous Spaces. Journal of Global Optimization, 11, 341359, 1997.

[22] De Cea, J., Fernández, E., Transit Assignment for Congested Public Transport Systems: An Equilibrium Model. Transportation Science, 27(2), 133-147, 1993.

[23] Chriqui, C., Robillard, P., Common Bu Lines. Transportation Science, 6(2), 115-121, 1975.

[24] Florian, M., Spiess, H., The convergence of diagonalization algorithms for asymmetric network equilibrium problems. Transportation Research Part B: Methodological, 16(6), 477-483, 1982.

[25] Miandoabchi, E., Farahani, R. Z., Szeto, W. Y., Bi-objective bimodal urban road network design using hybrid metaheuristics. Central European Journal of Operations Research, 20(4), 583-621, 2012.

[26] Sheffi. Y., Urban transportation networks, Prentice-Hall, NJ, USA, 1985.

[27] Frank, M., Wolfe, P., An algorithm for quadratic programming. Naval Research Logistics, 3(1-2), 95-110, 1956.

[28] Mandl, C. E., Evaluation and optimization of urban public transportation networks. European Journal of Operational Research, 5(6), 396-404, 1980.

[29] Arbex, R. O., da Cunha, C. B., Efficient transit network design and frequencies setting multi-objective optimization by alternating objective genetic algorithm. Transportation Research Part B: Methodological, 81(2), 355-376, 2015.

[30] Jha, S. B., Jha, J. K., Tiwari, M. K., A multi-objective meta-heuristic approach for transit network design and frequency setting problem in a bus transit system. Computers and Industrial Engineering, 130, 166-186, 2019. 